Supplementary Material for

\title{
A Gene Cluster Encoding Resistomycin Biosynthesis in Streptomyces resistomycificus: Exploring Polyketide Cyclization Beyond Linear and Angucyclic Patterns
}

Kathrin Jakobi \& Christian Hertweck*

Department of Bioorganic Synthesis Hans-Knöll-Institute for Natural Products Research, Jena, Germany 
1. Material and Methods

Bacterial Strains and Culture Conditions. Resistomycin producer Streptomyces resistomycificus HKI 237 was used as the source of DNA in the construction of the genomic DNA library. S. lividans TK23 ${ }^{10}$ served as host strain for heterologous expression experiments. For resistomycin production, wild-type and mutant strains were cultivated on R5 $\operatorname{agar}^{10}$ and M10 agar (yeast extract $4 \mathrm{~g} / \mathrm{l}$, malt extract $10 \mathrm{~g} / \mathrm{l}$, glucose $4 \mathrm{~g} / \mathrm{l}$, agar $20 \mathrm{~g} / \mathrm{l}, \mathrm{pH} 7.3$ ) for 5 days at $28^{\circ} \mathrm{C}$. Resistomycin production of the transformant $S$. lividans TK23/pKJ05 is at the wild type level. S. lividans TK23 was cultured on R5 agar and YEME liquid medium ${ }^{10}$ for protoplasts. Transformants were selected with thiostrepton (Sigma) at $25 \mu \mathrm{g} / \mathrm{ml}$ in solid and $2.5 \mu \mathrm{g} / \mathrm{ml}$ in liquid medium. E. coli strains EPI 100 (Epicentre) and strain XL1-Blue served as hosts for library construction and routine subcloning, respectively. E. coli strains were grown in LB medium supplemented with ampicillin $(100 \mu \mathrm{g} / \mathrm{ml})$ for selection.

Plasmids and General DNA Procedures. DNA isolation, plasmid preparation, restriction digests, gel electrophoresis and ligation reactions were conducted according to standard methods. ${ }^{10}$ pBluescript II SK(+) (Stratagene) was the routine vector for subcloning and preparation of DNA templates for sequencing. E. coli-Streptomyces shuttle vector pKJ01 was used for all expression experiments in Streptomyces. Restriction enzyme-digested DNA fragments were recovered from agarose gel by the GFX PCR DNA and Gel Band Purification Kit (Amersham).

Construction of $\boldsymbol{E}$. coli-Streptomyces shuttle vector. pKJ01 is a SCP2* derivative (pRM1), in which the $10.5 \mathrm{~kb} H i n \mathrm{dIII} / E c o$ RI fragment was replaced with a polylinker including a $c o s$ site. This cassette was obtained by subcloning a 1.13kb EcoRV / SspI cos fragment from pWEB (Epicentre) into the multicloning site (EcoRV) of pHGF7518 (T.-W. Yu, H.G. Floss, personal communication).

Library Construction and Screening. A S. resistomycificus genomic cosmid library was constructed in E. coli EPI 100 using pKJ01 as cosmid vector. Genomic DNA was sheared and size-fractionated by gel electrophoresis, yielding fragments with an average size between 35 and $40 \mathrm{~kb}$, and blunted using the end-repair mix (Epicentre). The entire library was pooled and introduced into S. lividans TK23 by PEG-mediated protoplast transformation. ${ }^{10}$ About 800 thiostrepton resistant transformants were phenotypically screened for the strong orange fluorescence of resistomycin at $366 \mathrm{~nm}$.

DNA Sequencing and Analysis. Cosmid pKJ05 was isolated from a positive transformant, sonicated, treated with BAL-31 nuclease (New England Biolabs), and end-repaired by the Klenow fragment (NEB). Size-fractionation by agarose gel electrophoresis yielded ca. $2 \mathrm{~kb}$ DNA fragments that were cloned into the EcoRV site of pBluescript II SK(-) for sequencing. Remaining gaps were filled by targeted subcloning and primer walking.

Isolation and Detection of Metabolites. Resistomycin was extracted from chopped agar plates and from liquid culture broths of wild-type and recombinant strains with ethyl acetate. Extracts were concentrated in vacuo after drying over $\mathrm{Na}_{2} \mathrm{SO}_{4}$ and redissolved in $\mathrm{MeOH}$. Resistomycin was identified by comparison of the extracts by thin-layer chromatography (TLC), high performance liquid chromatography (HPLC) and mass spectrometry (MS) with an authentic sample. Analytical HPLC runs were performed on a Shimadzu HPLC-system equipped with a Nucleosil100 C18 column $(5 \mu \mathrm{m}$ particle size, 125 x $3 \mathrm{~mm}$, GROM Analytik + HPLS GmbH). Metabolites were eluted with $\mathrm{MeCN} / 0.1 \%$ TFA in $\mathrm{H}_{2} \mathrm{O}$ gradient ranging from $0.5 \%$ to $99 \% \mathrm{MeCN}$ in $20 \mathrm{~min}$ at a flow rate of $1.0 \mathrm{ml} / \mathrm{min}$ and UV detection at $268 \mathrm{~nm}$. 
2. Genes of the rem cluster in alphabetical order, their deduced functions, corresponding protein sizes and closest homologs

\begin{tabular}{|c|c|c|c|c|c|c|}
\hline \multirow[t]{2}{*}{ Protein } & \multirow{2}{*}{$\begin{array}{l}\text { Size } \\
\text { [aa] }\end{array}$} & \multirow[t]{2}{*}{ Deduced Function } & \multicolumn{4}{|c|}{ Closest Homolog } \\
\hline & & & Protein & Origin & $\begin{array}{c}\text { Identities / } \\
\text { Positives } \\
{[\%]} \\
\end{array}$ & $\begin{array}{c}\text { Protein } \\
\text { Accession } \\
\text { Number } \\
\end{array}$ \\
\hline RemA & 428 & Ketosynthase KS $\alpha$ & RubA & S. collinus & $44 / 63$ & AAG03067 \\
\hline RemB & 412 & Ketosynthase KS $\beta$ & $\mathrm{TcmL}$ & S. glaucescens & $40 / 53$ & AAA67516 \\
\hline RemC & 83 & Acyl carrier protein & AcpP & S. coelicolor A3(2) & $38 / 57$ & CAA60201 \\
\hline RemD & 142 & PPTase & AcpS & S. avermitilis & 43 / 57 & BAC72676 \\
\hline RemE & 425 & $\begin{array}{l}\text { Malonyl-CoA:ACP } \\
\text { transacylase }\end{array}$ & StiB & Stigmatella aurantiaca & $37 / 51$ & CAD19086 \\
\hline RemF & 143 & Cyclase & $\mathrm{TcmJ}$ & S. glaucescens & $30 / 50$ & AAA67514 \\
\hline RemG & 349 & Methyltransferase & MtmMI & S. argillaceus & $28 / 45$ & AAD55585 \\
\hline RemH & 347 & Methyltransferase & GrhL & S. sp. JP95 & $24 / 41$ & AAM33664 \\
\hline RemI & 150 & Cyclase & $\mathrm{TcmN}$ & S. glaucescens & $39 / 52$ & S27696 \\
\hline RemJ & 335 & Kinase & ORF50 & S. rochei & $60 / 71$ & BAC76508 \\
\hline RemK & 160 & Unknown & RB11248 & Pirellula sp. & $34 / 42$ & CAD79012 \\
\hline RemL & 276 & Cyclase & ZhuJ & S. sp. 1128 & $41 / 55$ & AAG30196 \\
\hline RemM & 197 & TetR-family regulator & SAV5945 & S. avermitilis & $32 / 47$ & BAC73657 \\
\hline RemN & 515 & Efflux & SAV1847 & S. avermitilis & $42 / 58$ & BAC69558 \\
\hline $\mathrm{RemO}$ & 399 & FAD-dependent oxygenase & Atu2610 & Agrobacterium tumefaciens & $48 / 66$ & AAL43591 \\
\hline RemP & 221 & $\begin{array}{l}\text { Two-component response } \\
\text { regulator }\end{array}$ & SAV6671 & S. avermitilis & $35 / 53$ & BAC74382 \\
\hline RemQ & 195 & TetR-family regulator & SAV4715 & S. avermitilis & 43 / 61 & BAC72427 \\
\hline RemR & 149 & TetR-family regulator & SCM2.10 & S. coelicolor A3(2) & $28 / 23$ & CAB65638 \\
\hline
\end{tabular}

